\title{
Some time-delay finding measures and attractor reconstruction
}

\begin{abstract}
Topologically equivalent attractor reconstruction is one of the major issues in nonlinear analysis. This is because of the fact that the underlying dynamical model of some nonlinear phenomena may not be known and thus it is necessary to retrieve the dynamics from the data it generates. One way to achieve this is the reconstruction of the attractor. The basis of such reconstruction is the famous Taken's embedding theorem, which asserts that an equivalent phase space trajectory,preserving the topological structures of the original phase space trajectory, can be reconstructed by using only one observation of the time series. However, in some cases topologically equivalent attractor reconstructions can also be done by using multiple observations. All these things involve the choice of suitable time-delay(s) and embedding dimension. Various measures are available to find out the suitable time-delay(s). Among them, linear auto-correlation, Average mutual information, higher dimensional mutual information are mostly used measures for the reconstruction of the attractors. Every measures have certain limitations in the sense that they are not always useful in finding suitable time-delay(s). Thus it is necessary to introduce few more nonlinear measures, which may be useful if the aforesaid measures fail to produce suitable time-delay/time-delays. In this chapter, some comparatively new nonlinear measures namely generalized autocorrelation, Cross auto-correlation and a new type of nonlinear auto-correlation of bivariate data for finding suitable time-delay(s) have been discussed. To establish their usefulness, attractors of some known dynamical systems have been reconstructed from their solution components with suitable time-delay(s) obtained by each of the measures. These attractors are then compared with their corresponding original attractor by a shape distortion parameter Sd. This shape distortion parameter actually checks how much distorted the reconstructed attractor is from its corresponding original attractor. The main objective of this chapter is to address the problem of reconstruction of a least distorted topologically equivalent attractor. The reason is that if the reconstructed attractor is least distorted from its original one, the dynamics of the system can be retrieved more accurately from it. This would help in identifying the dynamics of the corresponding system, even when the dynamical model is not known. Out of the three measures discussed in this chapter, the generalized and cross autocorrelation measures produce least distorted topologically equivalent attractor only by consideration of multiple solution components of the dynamical system. On the other hand, by using the measure - new type of nonlinear auto-correlation of bivariate data, one can reconstruct a least distorted topologically attractor from single solution component of the dynamical system. Various numerical results on Lorenz system, Neuro-dynamical system and also on two real life signals are presented to prove the effectiveness of the aforesaid three comparatively new nonlinear time-delay finding measures. Finding of suitable embedding dimension is another important issue for attractor reconstruction. However, this issue has not been highlighted in this chapter because we have restricted this discussion only to three dimensional attractor reconstruction.
\end{abstract}

Keyword: Attractor; Non-linear; Parameter; Dynamics 